\title{
Resultados da fisioterapia hospitalar na função do membro superior comprometido após acidente vascular encefálico
}

\section{Results of physiotherapy in a hospital setting on upper limb function after stroke}

Suzana Corrêa França Valente ${ }^{1}$, Erica Bertáglia de Paula², Márcia Abranches 3 , Vanessa Costa ${ }^{4}$, Heloise Borges ${ }^{5}$, Therezinha Rosane Chamlian6, Danilo Masiero ${ }^{7}$

\section{RESUMO}

Objetivo: O objetivo deste estudo foi avaliar se a fisioterapia em ambiente hospitalar melhora a função do membro superior (MS) acometido em pacientes após acidente vascular encefálico, e os objetivos secundários foram avaliar a força muscular (FM) e a sensibilidade deste membro. Método: Participaram do estudo 8 pacientes com diagnóstico de AVE isquêmico. Estes receberam fisioterapia padronizada para o MS durante o período de internação no Hospital São Paulo, duas vezes por dia. Todos foram avaliados através das escalas de Fugl-Meyer modificada e de FM do Medical Research Council em dois momentos: antes e após o tratamento fisioterapêutico, a fim de obter dados comparativos de forma quantitativa em relação à função motora e sensibilidade, e força muscular do MS respectivamente. Resultados: Seis pacientes apresentaram melhora da força muscular e da função do MS, e cinco dos sete pacientes que apresentavam alteração da sensibilidade, apresentaram melhora após o tratamento fisioterápico. Conclusão: Desta forma a fisioterapia pode ser considerada de grande valia na reabilitação precoce de indivíduos hemiparéticos hospitalizados, proporcionando a estes melhora da função motora, sensibilidade e força muscular. No entanto os pacientes plégicos foram os menos beneficiados com o tratamento.

\section{Unitermos: Atividade Motora, Fisioterapia, Acidente Cerebrovascular.}

Citação: Valente SCF, Paula EB, Abranches M, Masiero D, Chamlian TR Chamlian, Lacanno SN, Borges H, Costa V. Resultados da fisioterapia hospitalar na função do membro superior comprometido após acidente vascular encefálico. Rev Neurocienc 2006; 14(3):122-126.

\section{SUMMARY}

Objective: The aim of this study was to analyze if the physiotherapy treatment in a hospital setting improves upper limb function post stroke. Methods: Eight patients who were diagnosed as having stroke and were admitted in Hospital Sao Paulo were included in this study. Each patient received physiotherapy treatment twice a day in this hospital. The upper limb physiotherapy treatment was standardized for all patients. The Fugl-Meyer scale modified was used to assess motor function. The quantitative analysis of this scale was performed comparing the

\footnotetext{
Trabalho realizado no Hospital São Paulo (HSP), São Paulo, SP.
}

1 - Fisioterapeuta da Fundação Selma, especializada em fisioterapia motora Hospitalar e Ambulatorial aplicada à neurologia pela UNIFESP e especializada em fisioterapia hospitalar pelo Hospital Israelita Albert Einstein.

2,3 - Fisioterapeuta do Hospital São Paulo e preceptora do curso de Fisioterapia motora hospitalar e ambulatorial aplicada à neurologia da UNIFESP.

4 - Fisioterapeuta especializada do Lar Escola São Francisco - LESF e preceptora do curso de Fisioterapia motora hospitalar e ambulatorial aplicada a neurologia da UNIFESP.

5 - Fisioterapeuta especializada do LESF, coordenadora e preceptora do curso de Fisioterapia motora hospitalar e ambulatorial aplicada à neurologia da UNIFESP.

6 - Chefe de clínica de fisiatria do departamento de ortopedia e traumatologia da UNIFESP e diretora técnica do LESF.

7 - Professor associado e chefe da disciplina de fisiatria do departamento de ortopedia e traumatologia da UNIFESP. 
total scoring points before and after treatment. Results: All patients demonstrated an improvement in function after the physiotherapy treatment, except for those patients who presented upper limb plegia. Conclusion: For this reason, physiotherapy treatment is a valuable tool to be use in the early rehabilitation process of hemiparetic patients after stroke.

\section{Keywords: Motor Activity, Physical Therapy, Cerebrovascular Accident.}

Citation: Valente SCF, Paula EB, Abranches M, Masiero D, Chamlian TR Chamlian, Lacanno SN, Borges H, Costa V. Results of physiotherapy in a hospital setting on upper limb function after stroke. Rev Neurocienc 2006; 14(3):122-126.

\section{INTRODUÇÃO}

O Acidente Vascular Encefálico (AVE) é o resultado da restrição sanguínea em determinada área do

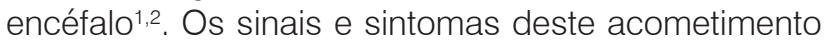
neurológico dependerão do local da lesão, sendo que vários tipos de comprometimento podem ser encontrados ${ }^{1,3-5}$. As atividades de vida diárias como alimentação, higiene e vestuário também serão prejudicadas, pela ausência ou diminuição da força e alteração do tônus muscular, além da presença de sinergismo patológico que dificultarão o movimento adequado do membro superior para a realização das mesmas s,3, 6,7. $^{2}$.

Os pacientes perdem algumas funções, e as recuperam (em parte ou quase totalmente) devido a dois componentes: o intrínseco, por recuperação neurológica anatômica e fisiológica, pela redução de edema cerebral, desenvolvimento de novas vias e plasticidade neuronal; e o adaptativo, que ocorre através do aprendizado de novas maneiras para executar as funções ${ }^{6}$. Sendo assim, a fisioterapia torna-se indispensável para a reorganização cortical e consequentemente o reaprendizado motor ${ }^{8,9}$. Algumas teorias acreditam que o tratamento precoce pode ser benéfico; de modo que previne alterações musculo-esqueléticas secundárias, como atrofia e dor, além de evitar o aprendizado de estratégias de movimentos anormais ou a inutilização do membro ${ }^{10-12}$. Além disso, há evidências de que a reabilitação do AVE reduz a incidência de complicações (pneumonia e outras complicações pulmonares, úlceras de decúbito e trombose venosa profunda), a taxa de mortalidade dos mesmos, e consequentemente o tempo de internação hospitalar ${ }^{13-15}$. Diante destes estudos realizados percebeu-se a grande importância de uma equipe de reabilitação hospitalar para auxiliar na recuperação precoce de pacientes pós AVE. Sendo assim, este trabalho teve como objetivo primário: verificar se a fisioterapia hospitalar promove melhora da função do membro superior plégico/parético após AVE; e como objetivos secundários: verificar aumento de força muscular (FM) do membro superior (MS) acometido destes indivíduos, e a melhora na sensibilidade do MS plégico/parético.

\section{MATERIAS E MÉTODOS}

Tipo de estudo: série de casos.

Local do estudo: Hospital São Paulo (HSP). São Paulo.
Comitê de Ética: O projeto foi enviado e aprovado pelo Comitê de Ética em Pesquisa.

Participantes:

Fatores de inclusão:

Os participantes do estudo apresentavam quadro motor de hemiparesia ou hemiplegia, por AVE isquêmico, internados nas enfermarias de neuroclínica e unidade de AVE do Hospital São Paulo no período de 15/07/2005 a 15/10/2005, independentes da data de alta hospitalar.

Fatores de exclusão:

- afasia; indivíduos com menos que 13 pontos na escala de Glasgow.

\section{Procedimentos e Método estatístico:}

Os pacientes foram avaliados pela autora principal do estudo, em dois momentos: logo que foram admitidos no Hospital São Paulo, e no dia da alta hospitalar, ou seja: antes e após a fisioterapia, para parâmetros de comparação de forma quantitativa. Desta forma, os dados foram analisados dicotomicamente: com melhora ou sem melhora.

Foram aplicados dois domínios da escala FuglMeyer modificada, já traduzida para o português ${ }^{16}$, para avaliação da função e sensibilidade no membro superior comprometido. A pontuação possível foi 66 pontos para a função motora da extremidade superior, e 12 pontos para a função sensorial. O aumento de cinco pontos no domínio de função motora corresponde a uma melhora estatisticamente significativa da função do membro superior destes pacientes ${ }^{17}$, e o aumento de um ponto no domínio de função sensorial significa melhora da sensibilidade ${ }^{9,18}$.

A força muscular do membro superior parético ou plégico também foi avaliada em dois momentos, conforme a escala de FM do Medical Research Council' ${ }^{19}$.

A conduta de tratamento para este membro foi padronizada e indiferenciada para os sujeitos do presente estudo.

Os participantes do estudo foram submetidos a atendimentos, realizados por fisioterapeutas treinados pela autora principal do estudo, a fim de realizá-los da forma mais uniforme possível. 
O tratamento fisioterapêutico foi realizado 2 vezes por dia, com duração aproximada de 25 minutos cada. Como o tratamento foi realizado durante o período de internação, a quantidade de terapias recebidas variou de paciente para paciente.

As diretrizes do tratamento realizado no membro superior acometido foram baseadas em princípios cinesioterapêuticos de livros, artigos científicos e de guidelines de fisioterapia ${ }^{1,7,10,20-27}$ e eram aplicáveis para todos os pacientes inclusos no estudo, independente do tônus muscular do membro superior. O tratamento realizado foi:

\section{Em decúbito dorsal:}

- Estimulação da sensibilidade do membro acometido, por aproximadamente 10 segundos com cada textura: algodão, lençol e luva de látex 20,27.

- Mobilização passiva do membro superior de forma lenta, iniciando pela mobilização de escápula em protração e elevação ${ }^{23,27}$, posteriormente da articulação da gleno-umeral, evoluindo para manuseios das articulações restantes (cotovelo, punho e dedos) ${ }^{1}$. Não existe um número de repetições ideal para cada paciente; e sim, uma quantidade adequada para alcançar o objetivo terapêutico de manutenção das amplitudes de movimento ${ }^{27}$; desta forma, foram realizadas as mobilizações passivas, com repetição mínima de 5 vezes em cada articulação.

- Alongamento do membro superior de forma passiva, com o membro com protração de escápula, rotação externa e abdução de ombro a aproximadamente $90^{\circ}$, extensão do cotovelo, supinação do antebraço e extensão de punho e dedos ${ }^{21,23,27}$. Esta posição era sustentada por quinze a trinta segundos, e repetida cinco vezes ${ }^{25}$.

- Na posição de alongamento citada acima, o terapeuta realiza estímulos lentos de aproximação articular a fim de potencializar aferências proprioceptivas dos músculos e articulações do membro superior ${ }^{1}$. Como cada paciente responde de forma individualizada às técnicas propostas, este manuseio deve ser realizado por cinco vezes, no mínimo ${ }^{25}$.

\section{Em decúbito dorsal elevado a noventa graus $\left(90^{\circ}\right)$ :}

- Auto manuseio de forma ativa ou assistida pelo terapeuta se necessário: a mão sadia segura a mão acometida, entrelaçando os dedos ou mantendo o antebraço em supinação, cotovelos estendidos, e ombros neutros (sem rotação); e realiza a flexo-extensão de ombros até 90 graus, para buscar a simetria corporal, além de alongamentos musculares 22,23,27.

- Treino da função, inicialmente de forma passiva e evoluindo para ativo assistido e por fim ativo livre, quando era possível o movimento adequado sem o auxílio do terapeuta. Foram enfatizadas combinações motoras que permitem a concretização de atividades funcionais do dia-a-dia ${ }^{1,23,27,28}$ :

a) levar a mão hemiparética à boca (simulando ou levando algum alimento ou copo) e retornar à posição de repouso de forma lenta;

b) levar a mão hemiparética até o topo da cabeça (simulando ou levando o pente de cabelo) e retornar à posição de repouso de forma lenta;

c) Levar a mão até a orelha contra-lateral e retornar a posição de repouso de forma lenta.

Os dois últimos exercícios foram repetidos, no mínimo 6 vezes, até que o paciente adquirisse melhor habilidade do movimento, ou seja, melhor velocidade e coordenação ${ }^{24,27}$ dentro de todas suas limitações motoras, com períodos de descanso se necessário.

Os pacientes foram orientados a concentrar-se na tarefa proposta e olhar para o membro superior ${ }^{1,7,20,27}$, e o terapeuta podia utilizar contatos manuais sempre que necessário $0^{1,12,23,29}$.

\section{RESULTADOS E DISCUSSÃO}

$\mathrm{Na}$ tabela 1 encontram-se as características dos participantes do estudo.

A amostra analisada foi de oito pacientes. A idade dos sujeitos variou de 40 a 81 anos. Este dado foi condizente com a literatura ${ }^{20}$ que comenta que o AVE geralmente ocorre a partir dos 65 anos, porém o número de jovens acometidos vem aumentando muito devido à exposição de fatores de risco, como contraceptivos orais, uso de drogas, fumo e estresse.

\begin{tabular}{l|c}
\hline Dados & Pacientes \\
\hline Sexo (valores brutos) & $\begin{array}{c}\text { Feminino: } 6 \\
\text { Masculino: } 2\end{array}$ \\
\hline $\begin{array}{l}\text { Idade em ano (Média e desvio } \\
\text { padrão) }\end{array}$ & 62,25 (13,76) \\
\hline Raça (valores brutos) & Negra: 1 \\
\hline $\begin{array}{l}\text { Parda: } 4 \\
\text { Topografia da lesão }\end{array}$ & $\begin{array}{c}\text { Artéria Cerebral Média: } 6 \\
\text { Artéria Cerebral Posterior: } 1 \\
\text { Artéria Cerebral anterior :1 }\end{array}$ \\
\hline $\begin{array}{l}\text { Tempo AVE em dias (Média e } \\
\text { Desvio padrão) }\end{array}$ & 5,62 (4,4) \\
\hline $\begin{array}{l}\text { Quadro motor do membro } \\
\text { superior (MS) (valores brutos) }\end{array}$ & Paresia de MS: 6 \\
\hline $\begin{array}{l}\text { Número de Atendimentos (Média } \\
\text { e desvio padrão) }\end{array}$ & Plegia de MS: 2 \\
\hline
\end{tabular}

Tabela 1. Características da amostra. 
A artéria acometida em $75 \%$ dos casos foi a cerebral média, que segundo alguns autores ${ }^{1}$ é o local mais comum para AVE e resulta em hemiparesia/plegia com predomínio braquial.

Em relação à conduta fisioterapêutica escolhida para a reabilitação destes pacientes, vale a pena ressaltar a importância da utilização das atividades do dia-a-dia, uma vez que os motiva à realização das tarefas, aumentando a atenção e facilitando o processamento das informações. Além disso, estas atividades habituais estimulam a memória e facilitam o reaprendizado motor ${ }^{1,7,20,27}$.

O tratamento visou ajudar os pacientes a moveremse do modo mais normal e com menor gasto energético possível, evitando que aprendam padrões anormais de movimento através da constante repetição ${ }^{23,29}$, desta forma, contatos manuais, podiam ser utilizados pelo fisioterapeuta na estimulação dos movimentos 1,12,23.

Quanto à melhora da função motora do membro superior após a fisioterapia (Gráfico 1), pudemos perceber que $75 \%$ da amostra, ou seja, 6 indivíduos apresentaram melhora na função motora do membro superior acometido. Os pacientes com quadro motor menos grave foram os mais beneficiados; sendo que os pacientes 3 e 8 , que encontravam-se com quadro motor de plegia em membro superior na primeira avaliação, não apresentaram melhora na função, ou seja, apresentaram aumento no domínio de função motora menor que 5 pontos. Em contrapartida, em 1998, alguns autores $^{9}$ observaram que o tratamento hospitalar para a melhora da função em membro superior foi mais efetivo em pacientes com déficit motor mais severo, no entanto os fatores de inclusão do artigo citado foram bastante rígidos, nos quais os pacientes deveriam ser capazes de levantar-se sozinhos e realizar o tratamento com independência (e supervisão) durante 30 minutos.

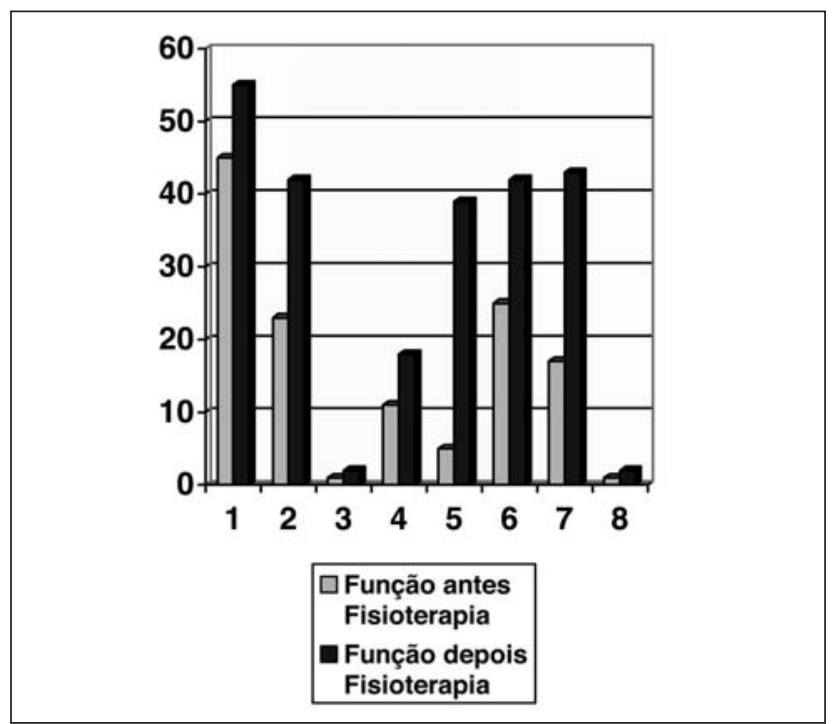

Gráfico 1. Comparação da função motora antes e após o tratamento fisioterapêutico. No eixo $X$ encontram-se os pacientes da amostra e no eixo Y a pontuação obtida através da escala Fugl-Meyer.
O número de atendimentos recebido pelos pacientes também devem ser discutidos, já que este número variou de indivíduo para indivíduo, dependendo do período de internação. Uma forte evidência encontrada neste estudo $^{12}$ concluiu que a fisioterapia melhora a função do membro superior de pacientes após um AVE, independente da freqüência. No estudo presente também não pode ser observada relação proporcional entre a quantidade de atendimentos e melhora na função motora; ou porque realmente não existe relação, ou porque muitos fatores como confusão, sonolência e déficit de atenção, tornam esta amostra bastante heterogênea, dificultando a comparação.

Em relação à sensibilidade (Gráfico 2), dos 8 pacientes inclusos no estudo, 7 apresentavam alteração de sensibilidade antes da fisioterapia, dos quais cinco $(71,42 \%)$ apresentaram melhora da sensibilidade, ou seja, beneficiaram-se através das atividades propostas.

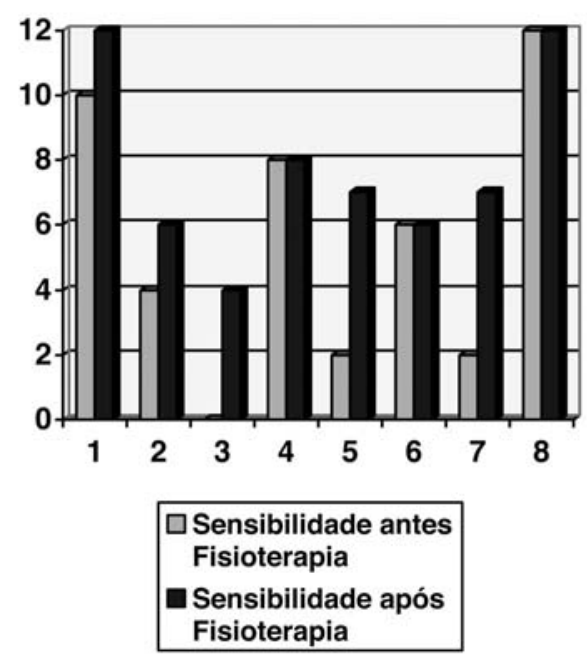

Gráfico 2. Comparação da sensibilidade antes e após o tratamento fisioterapêutico. No eixo $X$ encontram-se os pacientes da amostra e no eixo Y a pontuação obtida através da escala Fugl-Meyer.

O gráfico 3 demonstra o número de pacientes que apresentam aumento de força muscular, nos diferentes grupos musculares do MS acometido, após a fisioterapia. Todos os pacientes com quadro motor de paresia em membro superior apresentaram melhora da força muscular secundária ao aprimoramento da função. Apenas um paciente que apresentava plegia em MS obteve aumento de força muscular em abdutores de ombro, porém esta melhora não pode ser relacionada a um aumento de função.

Deve ser considerado o pequeno número de pacientes inclusos no estudo, uma vez que no período de três meses estipulado para a coleta de dados apenas estes oito pacientes internaram no HSP sob os critérios de inclusão para o estudo. 


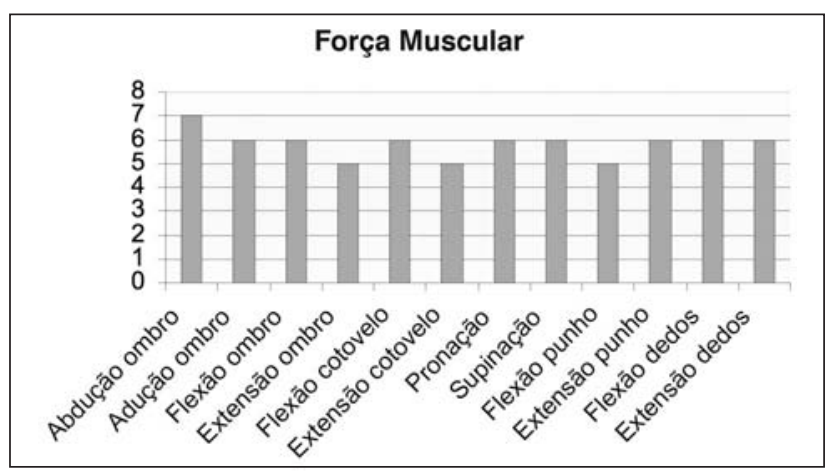

Gráfico 3. Número de pacientes que apresentaram aumento força muscular após o tratamento fisioterapêutico.

\section{CONCLUSÃO}

A fisioterapia pode ser considerada de grande valia na reabilitação precoce de indivíduos hemiparéticos hospitalizados por acidente vascular encefálico, proporcionando a estes melhora da função motora e sensibilidade, além de aumento de força muscular, independente da frequência, ou seja, número de atendimentos. Porém, os pacientes com quadro motor de plegia em membro superior não se beneficiaram com o tratamento proposto. Além disso, sugere-se a continuação deste estudo a fim de aumentar o número de participantes e gerar resultados mais conclusivos.

\section{REFERÊNCIAS BIBLIOGRÁFICAS}

1. O'Sullivan SB. Acidente Vascular Cerebral. In: O'Sullivan SB, Schmitz TJ. Fisioterapia: Avaliação e Tratamento. São Paulo: Manole, 1993, p. 385-421.

2. Barros JEF. Acidente Vascular Cerebral. In: Nitrini R, Bacheschi LA. A neurologia que todo médico deve saber. $4^{a}$ ed. São Paulo: Maltese, 1999, p. 133-166.

3. Smith LK, Weiss EL, Lehmkuhl LD. Aspectos de fisiologia muscular e neurofisiologia. In: Smith LK, Weiss EL, Lehmkuhl LD. Cinesiologia Clínica de Brunnstrom. $5^{\text {a }}$ ed. São Paulo: Manole, 1997, p. 139-142.

4. Sanvito LW. Síndromes Motoras Centrais. In: Sanvito LW. Propedêutica Neurológica Básica. São Paulo: Atheneu, 2000, p. 67-70.

5. Magri M, Silva NS, Nielsen MBP. Influência da inervação recíproca na recuperação da função motora de pacientes hemiplégicos por acidente vascular cerebral. Fisioter Bras 2003; 4(3): 223-226.

6. Laurito D, Bustamante M. La eficácia de un programa de rehabilitación en pacientes com accidente carebrovascular. Salus Militiae. 2001; 26(2): 85-88.

7. Carrié B. Bola suíça: teoria, exercícios básicos e aplicações clínicas. São Paulo: Manole; 1999. p 291-306.

8. Liepert J, Bauder H, Wolfgang HR, Miltner WH, Taub E, Weiller C. Treatmentinduced cortical reorganization after stroke in humans. Stroke 2000; 31 (6) 1210-1216.

9. Feys HM, Weerdt WJD, Selz BE, Stech GAC, Spichigner R, Vereeck LE et al. Effect of a Therapeutic Intervention for the Hemiplegic upper limb in the acute phase after stroke. A single blind, randomized, controlled multicenter trial. Stroke 1998; 29: 785-791.

10. Lauro AD, Pellegrino L, Savastano G, Ferraro C, Fusco M, Balzarano F et al. A randomized trial on the efficacy of intensive rehabilitation in the acute phase of ischemic stroke. J Neurol 2003; 250: 1206-1208.

11. Ottenbacher $\mathrm{KJ}$, Jannell $\mathrm{S}$. The results of clinical trials in stroke rehabilitation research. Arch neurol 1993; 50: 37-44.

12. Lincoln NB, Parry RH, Vass CD. Randomized Controlled Trial to evaluate Increased Intensity of Physiotherapy Treatment of Arm Function After Stroke. Stroke 1999; 30: 573-579.

13. Karla L, Dale P, Crome P. Improving Stroke Reabilitation: a controlled study Stroke 1993; 24 (10): 1462-1467.

14. Karla $L$. The influence of stroke unit rehabilitation on functional recovery from stroke. Stroke 1994; 25 (4): 821-825

15. Stevens RS, Ambler NR, Warren MD. A randomized controlled trial of a stroke rehabilitation ward. Age and Ageing. 1984; 13: 65-75.
16. Rizzo FV. Tradução e adaptação cultural da escala de avaliação da função motora Fugl-Meyer assessment (Tese). UNIFESP. 2004, 74p

17. Page SJ, Sisto SA, Levine P, Johnston MV, Hughes M. Modified constraint induced therapy: a randomized feasibility and efficacy study. $J$ Rehab Res Develop 2001; 38(5): 583-59018. Fugl-Meyer AR, Jaasko L, Leymann I, Olsson S, Steglin S. The post-stroke hemiplegic patient: a method of evaluation of physical performance. Scand J Reabil Med $1975 ; 7: 13-31$

19. Palmer ML, Epler ME. Princípios e técnicas de exames. In: Palmer ML, Epler ME. Fundamentos das técnicas de avaliação músculo esquelética. $2^{2}$ ed. Rio de Janeiro: Ganabara Koogan, 2000, p. 16-22.

20. Albuquerque SH. Acidente vascular encefálico. In: Teixeira E, Sauron FN, Santos LSB, Oliveira MC. Terapia Ocupacional na Reabilitação Física. São Paulo: Roca, 2003, p. 333-378.

21. Ryerson SD. Hemiplegia resultante de dano ou doença vascular. In: Umphered DA. Fisioterapia Neurológica. $2^{\mathrm{a}}$ ed. São Paulo: Manole, 1994, p. 615-654.

22. Horn Al, Fontes SV, Carvalho SM, Silvado RA, Barbosa PM, Durigan A Jr. et al. Cinesioterapia previne ombro doloroso em pacientes hemiplégicos/ paréticos na fase sub-aguda do acidente vascular encefálico. Arq NeuroPsiquiatr 2003, 613 (3B): 768-771.

23. Davies P. Encorajando o retorno da atividade no braço e mão e reduzindo o máximo de reações associadas. In: Davies P. Passos a seguir - um manual para o tratamento da hemiplegia no adulto. São Paulo: Manole, 1996, p. 128-154

24. Swenson JR. Exercício terapêutico na hemiplegia. In: Basmajian JV. Terapêutica por exercícios. São Paulo: Manole, 1987, p. 419-449.

25. Kisner C, Colby LA. Alongamento. In: Kisner C, Colby LA. Exercícios terapêuticos: fundamentos e técnicas. São Paulo: Manole, 1987, p. 125-126.

26. Edwards S, Patridge C, Mee R. Treatment schedules for research: a mode for physioterapy. Physioter 1990; 76: 605-607.

27. Cech D, Bohannon R, Byl N, Fincher K, Nicholson D, Potter K et al. Impaired motor function and sensory integrity associated with progressive disorders of the central nervous system. In: Rothstein JM. Guide to physical therapist practice. $2^{\mathrm{a}}$ ed. Phys Ther 2001; 81(1):375-392.

28. Woldag H, Hummelsheim H. Evidence-based Physiotherapeutic conceps for improving arm and function in stroke patients. J Neurol 2002; 249: 518-528.

29. Bobath B. Hemiplegia no adulto: Avaliação e Tratamento. São Paulo: Manole, 1978, p. 89-13. 